P148 (continued)

Design, Setting and Participants: We carried out a survey targeting 152 subjects of 5 to 12 years old, who enrolled in this study and visited community child centers located in Gyeongju, South Korea.

Outcome Measures and Analysis: SPSS program.

Results: Results showed significant differences in diverse side dishes of diversity realms, at 5 to 6 years old $(\mathrm{P}<$ 0.05). Also, regularity realms of NQ questionnaire showed significant differences by ages. In sodium intake habits, 4 items of 10 questions, that is preference of side dishes including a salted mackerel and dried shredded squid, preference of fried dish and fish cake with a soy sauce dip, frequency of a potato chip intake (over 3-4 times a week), and frequency of dine out/delivery food service (2-3 times a week, respectively), showed significant differences, according to age.

Conclusions and Implications: Nutrition education is necessary to increase the frequency of vegetable intake and reduce the frequency of sodium intake from food at community child care center. The current study will be foundational research for developing nutritional education programs for children in a community child center based on food behavior, nutritional status and sodium intake habits.

Funding: None.

\section{P149 Low-Income Pennsylvanian Parents of 10-14 Year Olds Reveal Stressors That Challenge Obesity Prevention Efforts}

Loren Archibeque, MPH, 1da126@psu.edu, Pennsylvania State University, 306 Chandlee University, Park, PA 16802; B. Lohse, PhD, RD, LDN

Objective: To describe the psychographics of a lower income, parent/caregiver population recruited to evaluate My Child's Weight, a program that addresses parent/caregiver concerns about their child's body size, development, and weight changes.

Design, Setting and Participants: Cross-sectional online survey (Qualtrics Pro, Provo, UT). Participants were parents/caregivers of children $10-14$ years old, recruited from low-income venues.

Outcome Measures and Analysis: Participants completed items from the SNAP-Education and Administration Reporting System, Satter Eating Competence Inventory, Three Factor Eating Questionnaire (TFEQR18), Sense of Coherence (3-item), Parent Modeling Behavior and Fruit and Vegetable Availability questionnaires along with items on weight perception and satisfaction, self-described eating disorders, food security, and the program evaluation. Data were analyzed using SPSS 22.0.

Results: Participants $(\mathrm{n}=30)$ were white, mostly female (93\%), lower income (90\%), 28 - 63 y; SNAP participants $(67 \%)$ with a profile of weight issues: Mean BMI was 34.7; 22 (73\%) were overweight/obese; 53\% were dissatisfied with their current weight, 30\% perceived current or previous eating disorder issues; only $27 \%$ were eating competent; $60 \%$ reported feeling stressed. Nearly all $(87 \%)$ were concerned about the weight of their $10-14$ $\mathrm{y}$ old children, but more so for parents dissatisfied with their own weight $(\mathrm{P}=0.011)$.

Conclusions and Implications: Findings suggest that developing successful childhood obesity prevention programs will need to address challenges presented by the high prevalence of parents/caregivers who are stressed, eating disordered and not eating competent. Consider parent vulnerabilities and weight-centered psychographics when developing and testing childhood obesity prevention interventions.

Funding: USDA, Supplemental Nutrition Assistance Program, Education.

\section{P150 Head Start Feeding Practices and Nutrition Trainings: Exploring the Needs of Teachers}

Meghan Fisher, MA, mfisher2@illinois.edu, University of Illinois at Urbana-Champaign, 1005 W. Nevada Street, Urbana, IL 61801; B. McBride, PhD; B. Fiese, PhD

Objective: Although previous research has identified effective approaches for professional development initiatives that target early childhood teachers, recent research has indicated that in-service training typically received by Head Start teachers short of evidence-based best practices. The current study explores Head Start teachers' views on current feeding practices and nutrition training, and identifies their training needs.

Design, Setting and Participants: Using phenomenological methods, the author completed 30 hours of observational and participatory fieldwork in each center that informed the creation of an interview protocol. Eleven Head Start teachers or teacher's aides from two different centers participated in one-on-one interviews. Additionally, participants completed a brief self-administered survey on their educational background and preferred training methods.

Outcome Measures and Analysis: A thematic analysis approach following guidelines outlined by Braun and Clarke (2006) was used. This involved read the data, generate initial codes, search for themes, review themes, define and name terms, and produce a report.

Results: The majority of teachers have had no formal nutrition education, and are relying solely on the trainings received through Head Start as their source of information on healthy feeding practices. Four main themes emerged from the interview data: variety of teachers' goals during mealtimes; views of current trainings; teachers' training needs that are not being addressed; and their preferred learning format.

Conclusions and Implications: The trainings received through Head Start initiatives are critical for the professional development of these teachers. Results from this exploratory study provide insight for the development of trainings that can impact teachers and promote the implementation of recommended nutrition related practices.

Funding: None. 